\title{
Modeling of detachment experiments at DIII-D
}

\author{
J.M. Canik ${ }^{\mathrm{a}^{*}}$, A.R. Briesemeister ${ }^{\mathrm{a}}$, C.J. Lasnier ${ }^{\mathrm{b}}$, A.W. Leonard ${ }^{\mathrm{c}}$, J.D. Lore ${ }^{\mathrm{a}}$, A.G. McLean ${ }^{\mathrm{b}}$, \\ and J.G. Watkins ${ }^{\mathrm{d}}$
}

${ }^{a}$ Oak Ridge National Laboratory, PO Box 2008, Oak Ridge, Tennessee 37831 USA

${ }^{b}$ Lawrence Livermore National Laboratory, 700 East Ave, Livermore, California 94550, USA

${ }^{c}$ General Atomics, PO Box 85608, San Diego, California 92186-5608, USA

${ }^{d}$ Sandia National Laboratories, 7011 East Ave, Livermore, California 94550 USA

\begin{abstract}
Edge fluid-plasma/kinetic-neutral modeling of well-diagnosed DIII-D experiments is performed in order to document in detail how well certain aspects of experimental measurements are reproduced within the model as the transition to detachment is approached. Results indicate, that at high densities near detachment onset, the poloidal temperature profile produced in the simulations agrees well with that measured in experiment. However, matching the heat flux in the model requires a significant increase in the radiated power compared to what is predicted using standard chemical sputtering rates. These results suggest that the model is adequate to predict the divertor temperature, provided that the discrepancy in radiated power level can be resolved.

Abstract length (150 words): 137 currently

PACS: 52.55.Fa, 52.55.Rk

PSI-21 Keywords: Divertor modelling, Detachment, SOLPS

*Corresponding author address: John Canik, Oak Ridge National Laboratory, PO Box 2008, MS-6169, Oak Ridge, TN 37831-6169, USA

*Corresponding and presenting author email: canikjm@ornl.gov

Presenting author: John Canik
\end{abstract}




\section{Introduction}

Future large fusion devices such as ITER require at least partially detached divertor operation in order to mitigate heat fluxes and ensure adequately long lifetimes of plasmafacing components (PFC) [1]. At present predicting the onset of detachment in such devices is based on fluid plasma models coupled to fluid or Monte Carlo neutral transport simulations, as embodied in such codes as SOLPS [2], UEDGE [3], EDGE2D-EIRENE [4] or SONIC [5]. However, these simulations are known to fail to capture several aspects of experiment, such as the divertor density and temperature [6], parallel flows and electric fields [7], and the processes involved in detachment itself [8-10]. Given that the partially detached operating point is based on projections using these same models, these shortcomings reduce confidence in the ability to actually achieve this state at the expected parameters. However, given the complex, highly coupled nature of the scrape-off-layer (SOL) and divertor system, identifying the underlying flaws in the model that give rise to these discrepancies is difficult.

In this work, a set of very well-diagnosed experiments performed in the DIII-D tokamak are analyzed. These experiments consist of a series of discharges with increasing lineaveraged density, obtained in L-mode operation, ranging in divertor conditions from strongly attached to fully detached. At each density, the position of the plasma was swept in the radial direction, so that fully 2D profiles of the electron density $\left(n_{\mathrm{e}}\right)$ and temperature $\left(T_{\mathrm{e}}\right)$ could be constructed based on measurements using the Divertor Thomson Scattering system (DTS) [11]. The ability to measure the 2D plasma profiles in this way, using a diagnostic that gives a reliable measurement (unlike Langmuir probes, where the interpretation is known to be difficult at low $T_{\mathrm{e}}[6]$ ), offers a unique opportunity to cross-check the plasma-neutrals models in detail to better determine which pieces of the model are failing to faithfully capture experiment and hence which might be the culprit behind some of the known problems. 


\section{Modeling approach}

The modeling performed in the present work uses the SOLPS5.0 suite of codes, which includes the B2.5 plasma fluid transport simulation [12], coupled to the EIRENE Monte Carlo neutral transport code [13] (using the so-called "1999" version of EIRENE). Transport in this model is assumed to be classical in the parallel direction (with corrections for kinetic effects), with ad-hoc user-specified transport coefficients for the cross-field direction. Deuterium and carbon are included in the simulations, carbon being generated using a constant chemical sputtering yield that in each separate case is set to the Davis-Haasz [14] value for the characteristic ion impact energy and PFC temperature. Physical sputtering is also included. The transport of hydrocarbons is not included in these simulations; the sputtered material is treated as atomic carbon.

Rather than address all aspects of detachment, such as the roll-over of the divertor ion flux as detachment progresses (which is known to be challenging to model [9]), we instead attempt to isolate and test smaller pieces of the model to assess their adequacy. In the work presented here, the component of the simulation being tested is the parallel transport model (particularly the parallel heat transport, although momentum and particle balance play roles as well). The basic question to be answered is: if the upstream density and temperature profiles are known (i.e., either taken from experiment or accurately predicted), as is the radial and parallel profile of the parallel heat flux, does the model accurately predict the divertor density and temperature? Several steps are taken to attempt to isolate this aspect. First only the outer divertor leg is considered; the inner divertor is still included in the simulations, but no additional effort is made to accurately reproduce the measured parameters in this leg. This raises the issue that the inner and outer divertor legs communicate with each other via, e.g., neutrals and drifts, potentially complicating this test if the inner divertor solution is inaccurate. Based on the simulations described here neutrals originating at the inner divertor 
contribute less than $5 \%$ of the electron cooling in the outer divertor, giving some confidence in conclusions drawn for the outer leg. These issues regarding the impact of inner divertor will be addressed in more detail in the future. The second step is to, at all points in any scans or steps performed, force the model to capture the upstream profiles as closely as possible. This is accomplished by adjusting the cross-field transport coefficients $D_{\perp}$ and $\chi_{\perp}^{\mathrm{i}, \mathrm{e}}$ in order to reproduce the experimental density and temperature profiles (which themselves come from well-constrained fits to the measurements). This can be done using established techniques [15], which are automated to remove user bias and inconsistency when judging the quality of the match. The third step is to force the simulation to reproduce the measured heat flux at the outer divertor. This can be accomplished by shifting the upstream profiles in the radial direction, such that $T_{\mathrm{e}}$ at the separatrix is varied; this is justified given the uncertainty in the exact position of the separatrix (shifts of $\sim$ several $\mathrm{mm}$ are sufficient to match the heat flux using this method). At present only the peak heat flux is directly constrained, although the radial profile turns out to agree reasonably well between simulation and experiment as will be shown. The final step is to force the code to give the measured value of the power radiated in the outer divertor. This is accomplished here by scaling the carbon radiation by a constant factor (in the present work, two cases are actually performed, one without this scaling factor applied and one with). With this final step taken, the parallel heat flux profile is as close to the experimental value as it can be made given the measurements, since the heat flux onto the target as well as the dominant volumetric losses are constrained. This allows the ability of the model to produce the measured divertor density and temperature to be evaluated. Additional terms will also affect the parallel heat flux profile, such as the poloidal dependence of crossfield transport and ion-electron collisional heat transfer (which is especially difficult to constrain without ion temperature measurements except for at the midplane). However, the impact of these can be at least tested later on by, for example, varying the ballooning-like 
nature of the cross-field transport coefficients. Likewise, the effect of $E \times B$ and diamagnetic drifts on this test of parallel transport can be assessed by repeating these steps without and with drifts. Here, due to numerical difficulties, only the case without drifts is addressed.

\section{Results of modeling}

The discharges modeled comprise a density scan in L-mode with a total of $\sim 1 \mathrm{MW}$ of power flowing into the SOL in all cases (assumed to be shared equally between electrons and ions at the core boundary of the simulations), with a lower single null shape and plasma current and toroidal magnetic field of 1.27 MA and -2.08 T, respectively. The line-averaged densities of the cases considered spanned the range $\left\langle n_{\mathrm{e}}\right\rangle=1.7-5.0 \times 10^{19} \mathrm{~m}^{-3}$, corresponding to fractions of the Greenwald density [16] of $f_{\mathrm{G}}=n / n_{\mathrm{G}}=0.15-0.45$. The divertor conditions range from strongly attached $\left(T_{\mathrm{e}} \sim 30 \mathrm{eV}\right)$ to fully detached $\left(T_{\mathrm{e}}<1 \mathrm{eV}\right)$. The PFC surface temperature in the divertor is $\sim 300-350 \mathrm{~K}$ in these discharges, with characteristic ion impact energies and chemical sputter yields based on [14] in the four cases considered of: $\mathrm{E}_{\mathrm{ion}}=100,45,17$, and $13 \mathrm{eV}$ and $\mathrm{Y}_{\text {chem }}=0.01,0.02,0.01$, and 0.01, respectively. At the main chamber wall, the characteristic impact energy is $30-60 \mathrm{eV}$ for the three lowest densities considered, and $\mathrm{Y}_{\text {chem }} \square=0.02$ is assumed; at the highest density the energy is reduced to $13 \mathrm{eV}$, and $\mathrm{Y}_{\text {chem }}=0.01$ is used in the modeling.

An example of the SOLPS fit to the measured upstream data for the lowest density case considered $\left(f_{\mathrm{G}}=0.15\right)$ is shown in Fig. 1, along with a comparison of the measured and modeled divertor profiles. The radially varying cross-field transport coefficients have values of $\mathrm{D} \sim 0.3 \mathrm{~m}^{2} / \mathrm{s}$ and $\chi_{\mathrm{e}, \mathrm{i}} \sim 2 \mathrm{~m}^{2} / \mathrm{s}$ near the separatrix (which are typical), and increase in the SOL similar to as is found in Refs 6 and 17; details of how these are adjusted to fit the measured upstream profiles can be found in Ref. 15. As the figure shows, for this strongly attached case, once the upstream profiles and peak divertor heat flux (measured by infrared 
thermography) are matched using the methods described above, the density and temperature profiles across the divertor floor show fair agreement with those measured from Langmuir probes and DTS. In this case the radiation is unscaled, and yields a total power radiated power from the outer divertor of $37 \mathrm{~kW}$, compared to $67 \mathrm{~kW}$ measured by bolometry. Scaling the radiation within SOLPS to agree with the measurements changes the resulting divertor profiles very little, since radiation is small compared to input power in either case.

The same comparison has been made for an intermediate density case $\left(f_{\mathrm{G}}=0.24\right)$ with $T_{\mathrm{e}} \sim 15-20 \mathrm{eV}$. In this case, SOLPS predicts a significantly hotter and more dense divertor plasma than is measured, with $n_{\mathrm{e}}$ and $T_{\mathrm{e}}$ off by a factor of $\sim 2-3$ both with and without the artificial radiation scaling (in this case the unscaled simulated divertor radiation is only $65 \mathrm{~kW}$, whereas $213 \mathrm{~kW}$ is measured). This is similar to results from modeling of ASDEX Upgrade low and intermediate collisionality discharges [6,17], and highlights the shortcomings of the fluid approximation at these parameters.

Here we focus instead on the high density and collisionality, nearly or fully detached cases that are of more interest for future reactor operation. Figure 2 shows the poloidal profile of $n_{e}$ and $T_{e}$ in a flux tube near the separatrix $\left(\psi_{N}=1.001-1.005\right)$, along with radial profiles along the divertor floor for the second highest density discharge modeled ( $f_{\mathrm{G}}=0.40$ ), which is nearly detached. Using the Davis-Haasz chemical sputtering yield, the model predicts a divertor $T_{\mathrm{e}}$ that is high compared to experiment, as is evident from both the poloidal and radial profiles (solid curves). The simulated radiated power in the outer divertor is also low in this case, being $\sim 160 \mathrm{~kW}$ whereas $\sim 300 \mathrm{~kW}$ are measured in experiment. With the carbon radiation scaled to match the bolometers, the simulated $T_{\mathrm{e}}$ is reduced near the divertor, resulting in much better agreement with DTS measurements (dashed curves). Similarly, $n_{\mathrm{e}}$ is increased in the simulation, agreeing better with experiment. 
Similar results are obtained for a discharge with a slightly higher line-averaged density $\left(f_{\mathrm{G}}=0.45\right)$, which shows a fully detached divertor. Without any artificial scaling of the radiated power, the power radiated in the outer divertor is nearly a factor of two lower than experiment (220 kW vs $400 \mathrm{~kW})$, and the divertor $\mathrm{T}_{\mathrm{e}}$ is higher than DTS measurements. With the radiated power accounted for, however, the poloidal temperature profile agrees quite well with DTS measurements, showing a broad poloidal region $(\sim 3 \mathrm{~cm})$ with $T_{\mathrm{e}}<3 \mathrm{eV}$ (Fig. 3). This level of agreement is an improvement on recent results using UEDGE to analyze DIII-D data [18], which typically showed difficulty in producing such a poloidally extended low $T_{\mathrm{e}}$ region. This may be due to the difficulty in getting sufficiently large radiated power levels in the simulation compared to experiment, which has also been observed using the UEDGE code when drifts are not included [19]. The agreement with the measured poloidal density profile is also good, except for a large localized $n_{\mathrm{e}}$ peak that is seen in the DTS measurements just above the divertor plate. This local maximum is seen across all densities examined, although not as pronounced as it is in this case. Figure 3 shows $2 \mathrm{D}$ profiles of the modeled and measured $n_{\mathrm{e}}$ and $T_{\mathrm{e}}$ distributions, which make the high density measured just above the floor evident. Further analysis is underway to determine if this is simply a calibration issue, or if SOLPS does indeed give such a large discrepancy in this region.

The results of the modeling naturally call into question the source of the factor of $\sim 2$ observed in the outer divertor radiation compared to experiment. One uncertainty is in the chemical sputtering yield assumed, which will directly affect the amount of carbon in the simulation and hence the radiation. To test this, a series of SOLPS runs has been repeated where the chemical yield was increased until the divertor radiation agreed with bolometers. The results are essentially unchanged from what has been presented so far, except that in this case the carbon emission can be compared to absolutely calibrated measurements. The comparison of CIII line radiation from simulation and from measurements using the 
Multichord Divertor Spectrometer [20] (MDS) is shown in Fig. 4, covering both the inner and outer divertors (with strike points at $\mathrm{R}=1.1 \mathrm{~m}$ and $\mathrm{R}=1.47 \mathrm{~m}$ ). As can be seen, in the strongly attached case the profile agrees well between model and measurement; the magnitude agrees with the unscaled Davis-Haasz yield case, with the increased yield case showing a factor of $\sim 2$ too large emission near the strike points. Although differences in the measured and modeled CIII profiles prevent a conclusive statement, the highest density detached case [Fig. 4(b)] also suggests that simply increasing the chemical sputtering yield results in more carbon being present in the machine than is supported by at least this spectroscopic measurement.

\section{Conclusions and future work}

The present work aims to test whether the parallel transport physics encapsulated within SOLPS is sufficient to describe the poloidal plasma profiles as measured by DTS (especially at the target), which is a is necessary piece in order to predict divertor detachment onset. For the high density cases of most interest, the answer appears to be affirmative for the parallel electron heat flux model (since the temperature profile is accurately captured), as long as the volumetric power losses are correctly taken into account. This is clearly a positive result, since the divertor temperature is a critical part of predicting the overall divertor behavior. However, correctly predicting the divertor radiation is also necessary, and this work has consistently shown a significant under-prediction (by a factor of approximately two); this is consistent with similar UEDGE modeling of DIII-D L-mode discharges. This motivates further research into the underlying cause of the low simulated radiated power; a first inspection suggests it is not simply due to an erroneously low chemical sputtering yield being assumed. Further comparisons of the code predictions to measurements of various radiating species are called for, especially for species expected to be the dominant radiators (i.e., 
Lyman- $\alpha$ and CIV [21]). It should also be noted that UEDGE work showed that activation of drifts in the simulation can significantly increase radiation [19], so it may be the case that the low simulated power here is simply due to the reduced physics setup used here, not a shortcoming of the full models used in codes such as SOLPS and UEDGE.

Addressing the role of drifts on assessing the parallel transport model will be a focus of future research. Conceptually this can be accomplished simply by repeating the steps above but with drifts activated; in practice this is challenging since drifts typically give rise to numerical difficulties (this is the reason for their exclusion to date). In addition, the sensitivity studies mentioned above (e.g., the effects of ballooning-like radial transport and uncertainty in the SOL ion temperature) will be performed to test the robustness of these results. An expanded set of discharges will also be examined, especially in H-mode since this is the regime anticipated in a reactor and also since a large effort has been undertaken recently $[22,23]$ to improve the prediction of the SOL heat flux width in this scenario. Finally, a clear need exists to test aspects of the parallel transport model beyond parallel electron heat flux. For example, the large density peak slightly removed from the divertor floor suggests a problem within the simulated momentum balance, which could be related for example to neutral recycling patterns. This aspect will be addressed in future experimental and modeling analysis.

\section{Acknowledgments}

Research supported by the US DOE under DE-AC05-00OR22725, DE-FC02-04ER54698, DEAC52-07NA27344, and DE-AC04-94AL85000. DIII-D data shown in this paper can be obtained in digital format following the link at https://fusion.gat.com/global/D3D_DMP. 


\section{References}

[1] A.S. Kukushkin, H.D.Pacher, Plasma Phys. Control. Fusion 44 (2002) 931.

[2] R. Schneider et al., Contrib. Plasma Phys. 46 (2006) 3.

[3] T. Rognlien et al., J. Nucl. Mater. 266-269 (1992) 654.

[4] A. Taroni et al., Contrib. Plasma Phys. 32 (1992) 438.

[5] K. Shimizu et al., Nucl. Fusion 49 (2009) 065028.

[6] A.V. Chankin et al., Plasma Phys. Control. Fusion 48 (2006) 839.

[7] S.K. Erents et al., Plasma Phys. Control. Fusion 46 (2004) 1757.

[8] G.D. Porter et al., Phys. Plasmas 3 (1996) 1967.

[9] M. Wischmeier et al., J. Nucl. Mater. 415 (2011) S523.

[10] K. Hoshino et al., J. Nucl. Mater. 415 (2011) S549.

[11] S.L. Allen et al., J. Nucl. Mater. 241-243 (1997) 595.

[12] B. Braams et al., Contrib. Plasma Phys. 36 (1996) 276.

[13] D. Reiter et al., Fusion Sci. Techol. 47 (2005) 172 and www.eirene.de

[14] J.W. Davis, A.A. Haasz, J. Nucl. Mater. 241-243 (1997) 37.

[15] J.M. Canik et al., J. Nucl. Mater. 415 (2011) S409.

[16] M. Greenwald et al., Nucl. Fusion 28 (1988) 2199.

[17] A.V. Chankin et al., Nucl. Fusion 49 (2009) 015004.

[18] M. Groth et al., "Poloidal distribution of recycling source and core fueling in DIII-D, AUG, and JET L-mode plasmas,” Proc. $38^{\text {th }}$ EPS Conf. on Control. Fusion and Plasma Physics, Strasbourg, 2011.

[19] M. Groth et al., J. Nucl. Mater. 415 (2011) S530.

[20] R.C. Isler et al., Phys. Plasmas 6 (1999) 1837.

[21] M.E. Fenstermacher et al., Phys. Plasmas 4 (1997) 1761.

[22] M.A. Makowski et al., Phys. Plasmas 19 (2012) 056112. 
[23] T. Eich et al., Nucl. Fusion 53 (2013) 093031. 


\section{Figure Captions}

Fig. 1. Radial profiles of the upstream (a) $n_{\mathrm{e}}$ and (b) $T_{\mathrm{e}}$, and divertor (c) heat flux as measured by IR camera, (d) parallel particle flux, (e) $n_{\mathrm{e}}$ and (f) $T_{\mathrm{e}}$ for a low- $n_{\mathrm{e}}$ discharge. Black lines are from SOLPS, red symbols are measured data (triangles indicate Thomson scattering data, squares Langmuir probes).

Fig. 2. Poloidal profiles along a near-separatrix flux-tube $\left(\psi_{\mathrm{N}}=1.001-1.005\right)$ for nearlydetached case of (a) $n_{\mathrm{e}}$ and (b) $T_{\mathrm{e}}$ (individual SOLPS curves for each grid cell within this range are shown); and radial divertor profiles of (c) $n_{\mathrm{e}}$ and (d) $T_{\mathrm{e}}$. Solid curves indicate SOLPS results without radiation scaling, dashed with scaling, and red symbols are Thomson scattering measurements.

Fig. 3. Poloidal profiles within a flux tube $\left(\psi_{N}=1.001-1.005\right)$ for fully-detached case of (a) $n_{\mathrm{e}}$ and (b) $T_{\mathrm{e}}$; 2D profiles of SOLPS (c) $T_{\mathrm{e}}$ and (d) $n_{\mathrm{e}}$, and measured (e) $T_{\mathrm{e}}$ and (f) $n_{\mathrm{e}}$. SOLPS results shown use scaled radiation (solid curves).

Fig. 4. Measured and simulated CIII emission for (a) low-density and (b) nearly-detached cases. Solid curves indicate SOLPS results with unscaled chemical sputtering, dashed with radiation increased to match radiated power from bolometers; red triangles indicate MDS measurements. 
Figure 3
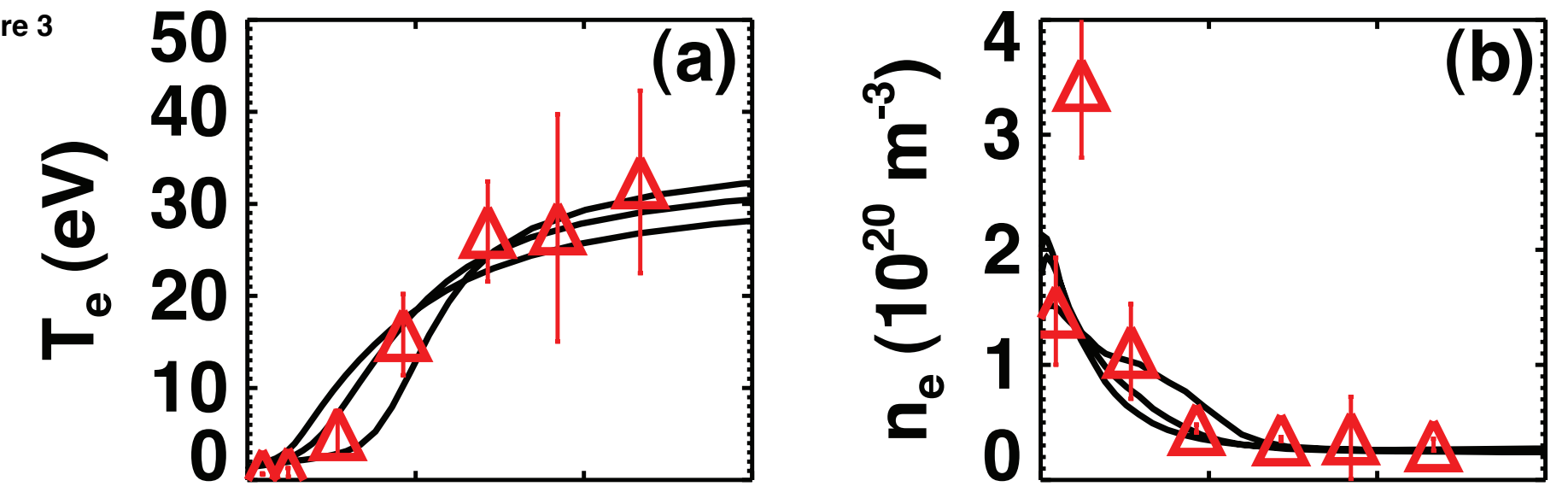

$\begin{array}{llll}0.0 & 0.1 & 0.2 & 0.3\end{array}$

$$
\begin{gathered}
\mathrm{L}_{\text {pol }}(\mathrm{m}) \\
\mathrm{T}_{\mathrm{e}}(\mathrm{eV})
\end{gathered}
$$

$\begin{array}{llll}0.0 & 0.1 & 0.2 & 0.3\end{array}$

$\mathrm{L}_{\text {pol }}(\mathrm{m})$

$\mathrm{n}_{\mathrm{e}}\left(10^{20} \mathrm{~m}^{-3}\right)$
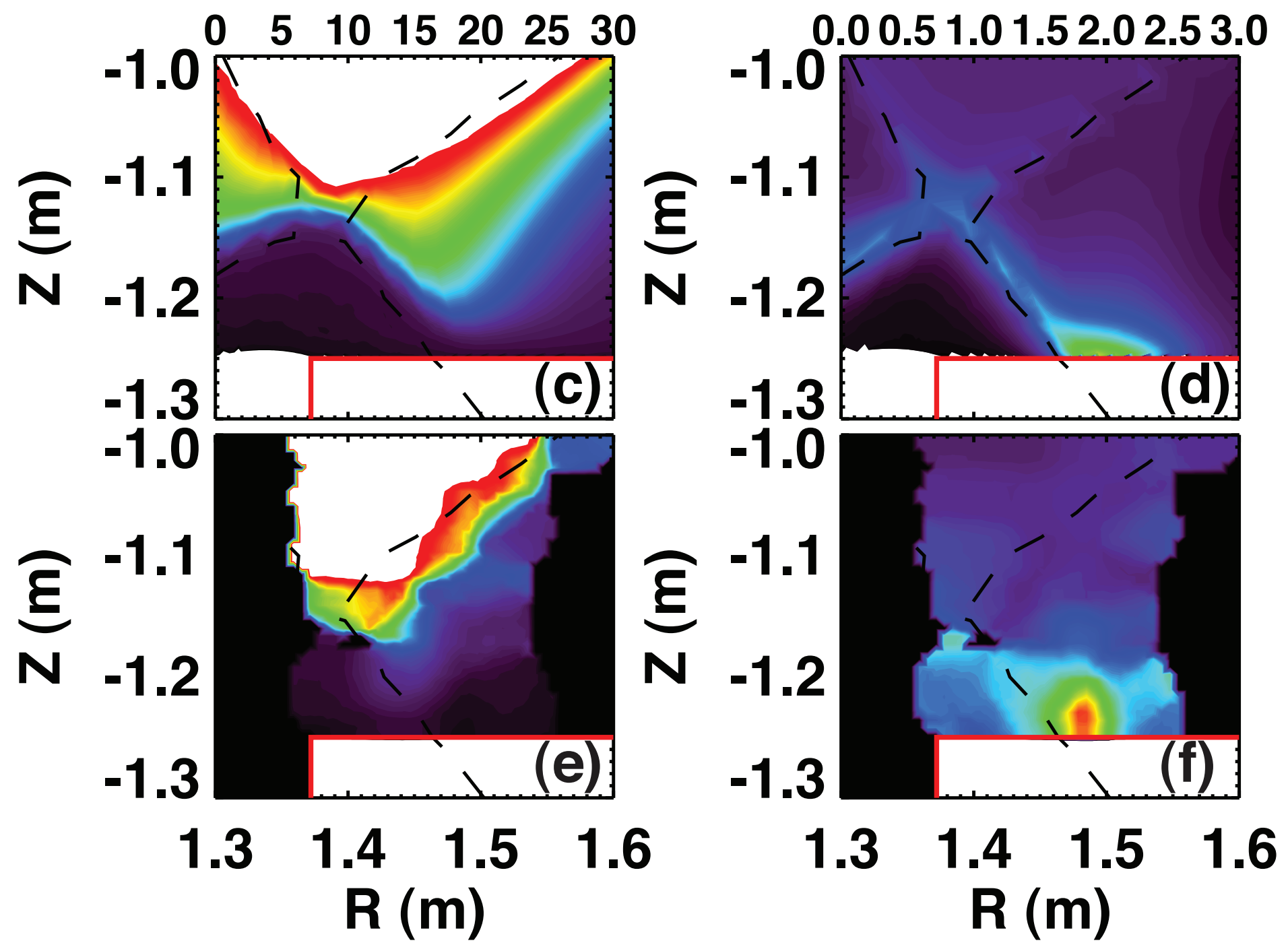

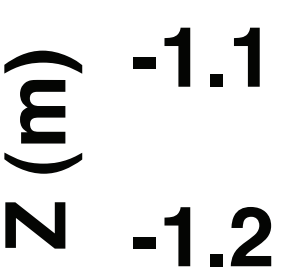

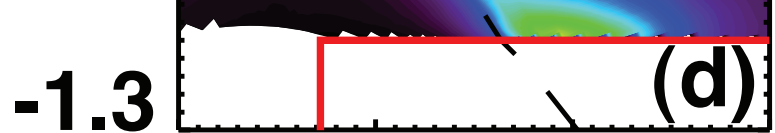

$-1.0$

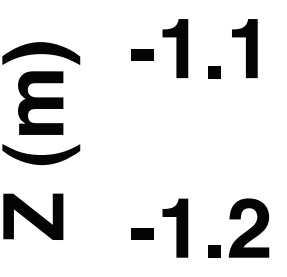

$-1.3$

(f)

$\begin{array}{llll}1.3 & 1.4 & 1.5 & 1.6\end{array}$

R (m) 


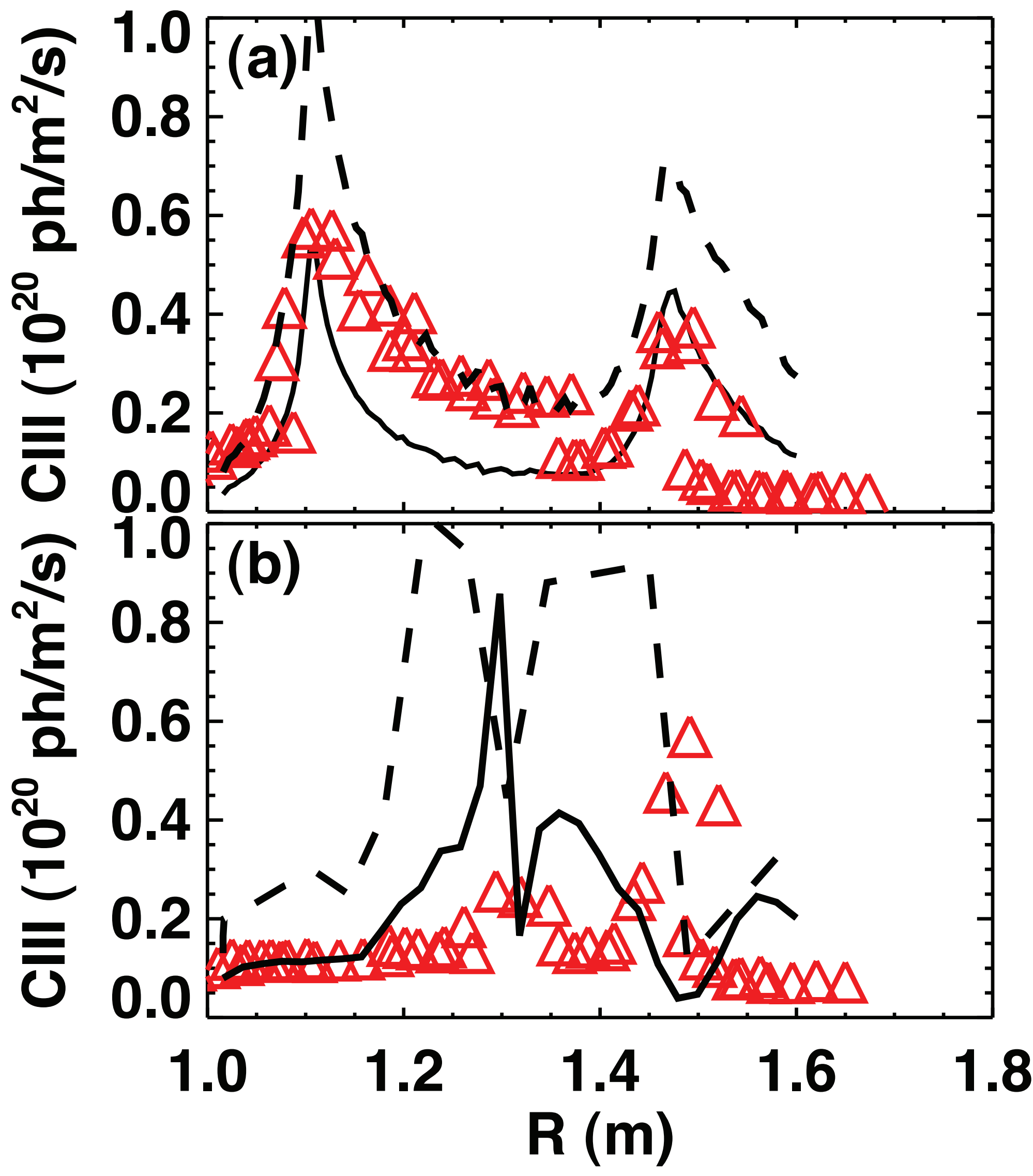

J.M. Canik 